\title{
Forgotten Perinephric Drain Anchored in the Tissues: Lessons to Be Learned
}

\author{
Prashant Kumar, MBBS, MS, and Rishi Nayyar, MBBS, MS, MCh
}

\begin{abstract}
Background: Drainage of any deep seated abscess often requires placement of catheters along with other conservative measures. These catheters are removed when the drainage volume reduces to clinically insignificant levels. However, if left in situ, there are potential complications. One such complication necessitating additional surgical procedure is highlighted in this case report. Malecot's catheter and children may be more at risk for such a complication.

Case Presentation: A 7-year-old girl presented with recurrent episodes of right flank pain associated with highgrade fever with chills for the last 5 months and right perinephric drain in situ. She had earlier presented at an outside center and was found to have bilateral renal calculi and left lower ureteral calculi along with right perinephric abscess and pyonephrosis. She underwent right perinephric drain and bilateral Double J (DJ) placement 4 months ago. The perinephric drain initially drained around $250 \mathrm{~mL}$ pus each day and progressively ceased to drain by 15-20 days. However, the drain was left in situ and the girl was referred for management of bilateral renal and left ureteral calculi. Pending her consultation, the drain and stents remained forgotten. At presentation, blood urea and serum creatinine were 20 and $0.2 \mathrm{mg} \%$, respectively. Urine culture was sterile. Non-contrast computerized tomography kidney, ureter, and bladder radiograph showed right perinephric drain, bilateral DJ stents with bilateral renal (lower and middle caliceal) calculi, and a chain of left upper ureteral calculi. A small loculated subcapsular collection was also noted at the lower pole of right kidney. All efforts made to pull out the drain under local anesthesia were in vain. The drain was found to be impacted and could not be taken out. Decision was taken to remove the drain laparoscopically. Drain was removed effectively and $\mathrm{B} / \mathrm{l}$ DJ stents were changed followed by staged procedure for calculi.

Conclusion: Malecot catheters may be more prone to ingrowth of tissue because of their inherent design of wider holes, all located at the tip of the catheter. This unique case emphasizes the need for careful follow-up of a patient with perinephric drain and difficulties with the removal of a Malecot catheter compared with a pigtail catheter, particularly in children. Laparoscopic removal of retained Malecot catheter as perinephric drain is a safe option of treatment in such a case.
\end{abstract}

Keywords: forgotten perinephric drain, Malecot catheter, laparoscopic, fragmentation of impacted catheter

\section{Introduction}

$\mathbf{P}$ ERINEPHRIC DRAIN PLACED PERCUTANEOUSLY is an effective method of draining perinephric abscess. Malecot's catheter has been traditionally used for this purpose. A forgotten Malecot catheter can become impacted due to tissue ingrowth into the holes in the drain. We present laparoscopic management in such a case for removal of impacted drain anchored in the tissues.

\section{Case Presentation}

A 7-year-old girl presented with recurrent episodes of right flank pain associated with high-grade fever with chills for the last 5 months and right perinephric drain in situ. She had earlier presented at an outside center and was found to have bilateral renal calculi and left lower ureteral calculi along with right perinephric abscess and pyonephrosis. She underwent right perinephric drain and bilateral Double J

Department of Urology, AIIMS, New Delhi, New Delhi, India.

(C) Prashant Kumar and Rishi Nayyar 2017; Published by Mary Ann Liebert, Inc. This is an Open Access article distributed under the terms of the Creative Commons Attribution License, which permits unrestricted use, distribution, and reproduction in any medium, provided the original work is properly cited. 
FIG. 1. X-ray kidney, ureter, and bladder radiograph (A) and NCCT (B) showing right perinephric drain, bilateral DJ stents with bilateral renal (lower and middle caliceal) calculi, and a chain of left upper ureteral calculi. A small loculated subcapsular collection can also be noted at the lower pole of right kidney. DJ, Double J; NCCT, non-computerized contrast tomography.
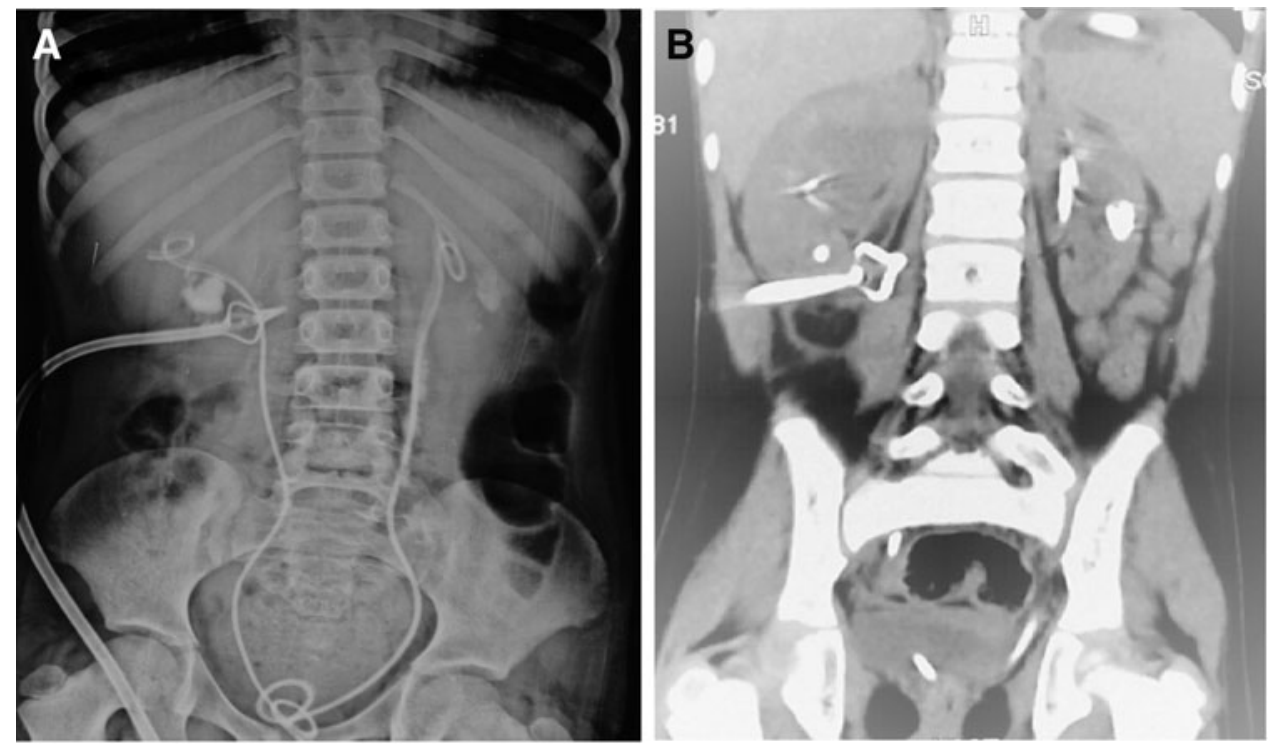

placement 4 months ago. The perinephric drain initially drained around $250 \mathrm{~mL}$ pus each day and progressively ceased to drain by 15-20 days. However, the drain was left in situ and the girl was referred for management of bilateral renal and left ureteral calculi. Pending her consultation, the drain and stents remained forgotten. At presentation, blood urea and serum creatinine were 20 and $0.2 \mathrm{mg} \%$, respectively. Urine culture was sterile. Imaging findings are shown in Figure 1. Differential renal function on LLEC renal dynamic scan was $82 \%$ and $18 \%$ on the right and left side, respectively. All efforts made to pull out the drain under local anesthesia were in vain. The drain was found to be impacted and could not be taken out. Given its treacherous location with tip sitting near the inferior vena cava (IVC), upper ureter, and lower pole of kidney, decision was taken to remove drain laparoscopically under vision with general anesthesia. Three ports $(12,5$, and $5 \mathrm{~mm})$ were used. After reflecting mesocolon, the drain was found to be impacted due to ingrowth of granulation tissue into the eyes of the Malecot catheter. Dense fibrotic reaction in and around the catheter necessitated sharp and careful dissection to save surrounding structures. The hepatic flexure of colon was also pulled up into the scar tissue (Fig. 2). Intraoperative blood loss was minimal. Bilateral DJ stents were changed. Patient had an uneventful recovery. Right percutaneous nephrolithotomy (PCNL), followed by left ureteroscopy and PCNL, was done subsequently as a staged procedure with complete clearance. Metabolic workup for stone disease was also done and normal. At the 6-month follow-up, the patient remained symptom free.

\section{Discussion and Literature Review}

Perinephric drain placed percutaneously is an effective method of draining perinephric abscess. Perinephric drains are usually either pigtail catheters or Malecot catheters with wide side holes. The external surface of pigtail catheters is smooth and most of the times easily removed. However, as evidenced in this case, there is a potential of tissue ingrowth into the holes in the drain. This is a disastrous iatrogenic complication, which is completely avoidable by not keeping the drain beyond its clinical utility. Children with their capacity for rapid healing might be especially prone to such a complication leading to entrapped drain tube necessitating additional anesthesia and surgical risk. Holes in any drainage tube are prone to ingrowth of granulation tissue. Because of large bore size of the hole and all four holes located at the same point near the tip, a Malecot-type tip is theoretically more prone to tissue ingrowth, which has the potential to meet inside the catheter to form a tissue bridge running across through the interior of the catheter. We encountered such a
FIG. 2. Intra-abdominal part of the polyurethane Malecot's catheter (perinephric drain) retrieved after transperitoneal laparoscopic intervention. The flanges of the tip were circumferentially embedded with the ingrowth of granulation and fibrotic tissue into the lumen of the catheter.
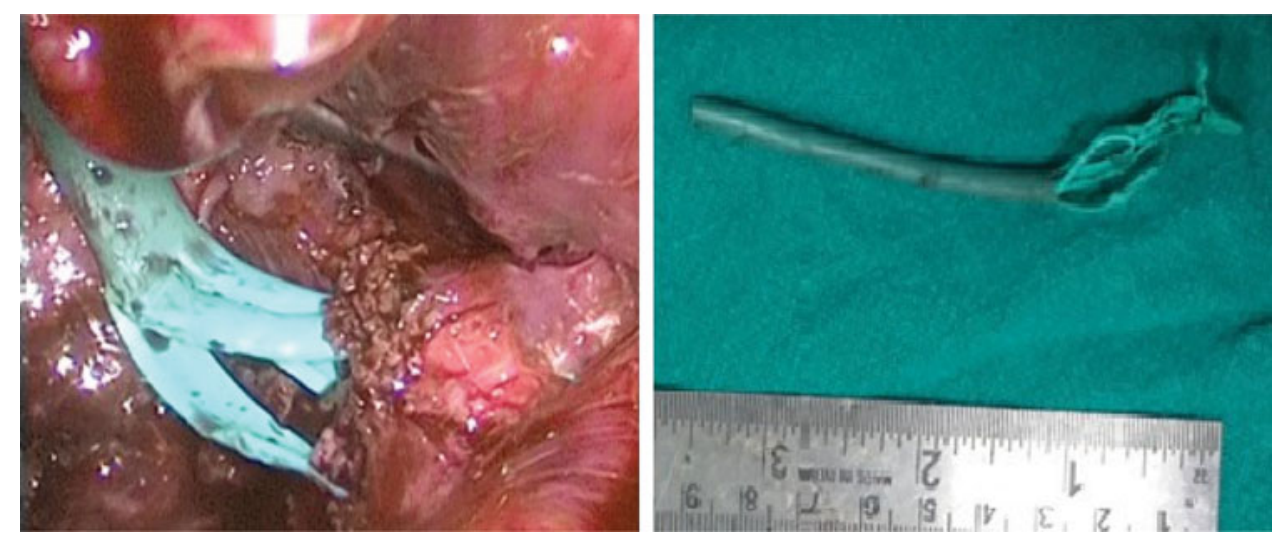
complication in our case. Although the time duration for which the drain was left in situ was certainly a factor, predisposition due to the use of a Malecot-type catheter cannot be ruled out. Besides, drains are known to erode into surrounding tissues. Risk factors for viscus injury are rigid catheters and chronic inflammatory conditions. Erosion of suprapubic catheter tubes into bowel is well known. ${ }^{1}$ Fragmentation of catheter is also known and may occur in the process of removal of a fixed catheter. ${ }^{2}$ In this particular study, the flower of the Malecot catheter broke during removal on one side following bilateral pyeloplasty. Open pyelotomy was required for removal. Retained catheters left for a long time are prone to infection.

In this particular case, perinephric drain was seen on contrast enhanced computerized tomography to be traversing through the parenchyma and forceful removal of catheter could have led to renal parenchymal tear and inadvertent bleeding, duodenal or colonic tear, or even IVC tear, besides a possibility of breakage of catheter. Pyelolithotomy/nephrolithotomy was not considered at the time of drain removal because of the location of major stone bulk in the lower calix, leaving it for PCNL as a staged procedure.

Nephrostomy tubes occasionally are resistant to extraction. ${ }^{3}$ Malecot nephrostomy tubes should be used with caution for long-term drainage of small intrarenal pelvis. In this particular study, the entrapped nephrostomy tubes were removed by endoscopically incising an anchoring tissue bridge that had grown over a flange. A small endoscope was advanced through the lumen of the entrapped catheter to allow for adequate visualization and electrocautery of the anchoring tissue bridge. Laparoscopic removal of retained nephrostomy tube has earlier been described. ${ }^{4}$ In this particular study, an initial ureteroscopic attempt at removal was unsuccessful, since it was not possible to grasp the tube adequately with sufficient torque. Intraoperatively, the tip of the nephrostomy tube was identified in the retroperitoneal space. The broken tip was freed from the fibrous tissue and retained tube was then removed laparoscopically in its entirety and without technical difficulty. Percutaneous approach was not considered in our case because half of the tube was lying outside the pelvicaliceal system and safe percutaneous access to the broken end looked difficult because of the proximity to the colon. There have been reported cases of nephrostomy tubes resistant to removal, but a retained perinephric drain has rarely been seen.

\section{Conclusion}

This unique case emphasizes the need for careful followup of a patient with perinephric drain and difficulties with the removal of a Malecot's catheter compared with pigtail catheter, particularly in children. Laparoscopic removal of a retained Malecot catheter as perinephric drain is a safe option of treatment in such a case.

\section{Disclosure Statement}

No competing financial interests exist.

\section{References}

1. Kass-Iliyya A, Morgan K, Beck R, et al. Bowel injury after a routine change of suprapubic catheter. BMJ Case Rep 2012; 2012:bcr2012006524.

2. Garg RK, Menon P, Narasimha Rao KL, et al. Pyeloplasty for hydronephrosis: Issues of double $\mathrm{J}$ stent versus nephrostomy tube as drainage technique. J Indian Assoc Pediatr Surg 2015;20:32-36.

3. Sardina JI, Bolton D, Stoller ML, et al. Entrapped Malecot nephrostomy tube: Etiology and management. J Urol 1995; 153:1882-1883.

4. Biyani CS, O'Connor JP, Browning AJ, et al. Laparoscopic removal of retained nephrostomy tubing. J Urol 2003;170: 916-917.

Address correspondence to:

Prashant Kumar, MBBS, MS

Department of Urology

AIIMS, New Delhi

New Delhi 110029

India

E-mail: prashant.su@gmail.com

$$
\begin{aligned}
& \text { Abbreviations Used } \\
\text { DJ } & =\text { Double } \mathrm{J} \\
\text { IVC } & =\text { inferior vena cava } \\
\text { LLEC } & =1 \text {-ethyl-cysteine } \\
\text { PCNL }= & \text { percutaneous nephrolithotomy }
\end{aligned}
$$

Cite this article as: Kumar P, Nayyar R (2017) Forgotten perinephric drain anchored in the tissues: Lessons to be learned, Journal of Endourology Case Reports 3:1, 123-125, DOI: 10.1089/cren.2017.0067. 